\title{
Eculizumab: A Review in Generalized Myasthenia Gravis
}

\author{
Sohita Dhillon ${ }^{1}$
}

Published online: 12 February 2018

(C) Springer International Publishing AG, part of Springer Nature 2018

\begin{abstract}
The humanized monoclonal antibody eculizumab $\left(\right.$ Soliris $\left.{ }^{\circledR}\right)$ is a complement inhibitor indicated for use in antiacetylcholine receptor (AChR) antibody-positive adults with generalized myasthenia gravis (gMG) in the USA, refractory gMG in the EU, or gMG with symptoms that are difficult to control with high-dose IVIg therapy or PLEX in Japan. It is the first complement inhibitor to be approved for use in these patients. In the well-designed, 26-week REGAIN study in patients with anti-AChR-positive refractory gMG, although a statistically significant benefit of eculizumab over placebo in the prespecified primary endpoint analysis (change from baseline in MG-activities of daily living (ADL) score assessed by worst-rank ANCOVA) was not formally demonstrated, preplanned and post hoc sensitivity analyses of this outcome, as well as other secondary outcomes supported the efficacy of eculizumab. Overall, patients receiving eculizumab experienced significant improvements in the ADL, muscle strength and healthrelated quality of life (HR-QOL) parameters relative to patients receiving placebo. Moreover, an ongoing extension of REGAIN showed that treatment benefits with eculizumab were sustained during continued therapy for at least 52 weeks. Eculizumab was generally well tolerated in these studies, with a tolerability profile similar to that reported
\end{abstract}

The manuscript was reviewed by: $S$. Berrih-Aknin, Sorbonne Université, INSERM, Association Institut de Myologie, Centre de Recherche en Myologie, UMRS974, Paris, France; K. Utsugisawa, Department of Neurology, Hanamaki General Hospital, Hanamaki, Japan.

Sohita Dhillon

demail@springer.com

1 Springer, Private Bag 65901, Mairangi Bay, Auckland 0754, New Zealand previously in other indications. Although several questions remain, such as duration of treatment, cost effectiveness and long-term efficacy and tolerability, current evidence indicates that eculizumab is a valuable emerging therapy for patients with refractory gMG.

Eculizumab: clinical considerations in anti-AChR antibody-positive gMG

Binds to C5 complement protein and inhibits the activation of terminal complement, thereby protecting the neuromuscular junction from the destructive effects of antibody-mediated complement activation

Significantly improved the ADL, muscle strength and HR-QOL relative to placebo in secondary analyses of the pivotal REGAIN study in patients with refractory disease, but did not achieve statistical significance in the prespecified primary endpoint analysis

Treatment benefits maintained for up to at least 52 weeks in an ongoing extension study

Generally well tolerated, with most adverse events of mild or moderate severity; headache is the most frequently reported adverse event

\section{Introduction}

Myasthenia gravis (MG), is an antibody-mediated, chronic autoimmune disease of neuromuscular synaptic transmission [1, 2], occurring at an incidence of 1.7-21.3 cases per million person-years during 1950-2007 [3]. It is 
characterized by fluctuating muscle weakness and fatigue, caused by antibodies directed against skeletal muscle receptors and proteins at the neuromuscular junction (NMJ) $[4,5]$. Symptoms of MG may include impaired speech, difficulty swallowing or chewing, shortness of breath, drooping of one or both eyelids, blurred vision and weakness in limbs, which have considerable impact on healthrelated quality of life (HR-QOL) [6].

MG can be classified based on: pathogenic antibody specificity [anti-acetylcholine receptor (AChR) antibodypositive, anti-muscle-specific tyrosine kinase (MuSK) antibody-positive, anti-low-density lipoprotein receptorrelated protein 4 (LRP4)-positive or seronegative]; thymus histology (thymitis, thymoma or atrophy), age of onset (before puberty, $<50$ or $>50$ years) and extent of involvement (ocular or generalized) [7]. The majority $(\approx 85 \%)$ of patients with MG have circulating antibodies against AChRs [5]. Binding of these anti-AChR antibodies to complement factors induces the formation of membrane attack complexes (MAC), which damage the muscle membrane and reduce the number of functional AChRs, leading to impaired neurotransmission $[5,8]$.

Treatment strategies for MG include: (1) improving neuromuscular transmission with the use of acetylcholinesterase inhibitors (e.g. pyridostigmine), (2) treatment of acute exacerbations [e.g. plasma exchange (PLEX), intravenous immunoglobulin (IVIg)], (3) immunosuppressive therapy (IST; e.g. corticosteroids, azathioprine, cyclosporine) and (4) thymectomy [7]. However, of these, only acetylcholinesterase inhibitors (e.g. pyridostigmine) are specifically indicated for use in patients with MG. Patients with MG are often treated successfully with these therapies and live productive lives; however, despite improved life-expectancy with advances in medical therapy, quality of life remains reduced, particularly in patients with generalized symptoms or active disease [9]. Moreover, a small subgroup of patients have treatment-refractory disease and require more aggressive therapy to prevent life-threatening crises [10]. Although its exact prevalence is unknown, treatment-refractory MG is estimated to affect $\approx 10-15 \%$ of patients with generalized MG (gMG) [10, 11]. Treatment options for these patients include chronic PLEX, chronic IVIg, rituximab and cyclophosphamide [12]. However, until recently, there were no options for patients who were refractory to currently available therapies, indicating an unmet need for additional therapies.

Complement plays a key role in the pathogenesis of disease. In clinical studies in patients with MG, IgG and C3 colocalized on the segments of the postsynaptic membrane and on fragments of degenerating junctional folds in the synaptic space [13] and MACs were detected at the muscle end-plates [14]. Moreover, in experimental autoimmune
MG (EMAG) studies, inhibition of complement pathways reduced MAC deposits at the NMJ, thereby diminishing the destruction of the NMJ by anti-AChR antibodies [1, 15]. Based on these observations, it was hypothesized that inhibition of complement activation in patients who have anti-AChR antibodies could prevent damage at the postsynaptic membrane of the NMJ, providing a rationale for the development of complement inhibitors.

Eculizumab $\left(\right.$ Soliris ${ }^{\circledR}$ ) is a recombinant humanized monoclonal $\mathrm{IgG} 2 / 4_{\mathrm{K}}$ antibody that binds to human C5 complement protein and inhibits the activation of terminal complement [16, 17]. It is the first targeted complement inhibitor approved worldwide for the treatment of complement-mediated diseases, including paroxysmal nocturnal haemoglobinuria (PNH) and atypical haemolytic uremic syndrome (aHUS). Recently, eculizumab was also approved for the treatment of adults with anti-AChR antibody-positive gMG in the USA [16], anti-AChR antibody-positive refractory $\mathrm{gMG}$ in the EU [17] or patients with anti-AChR antibody-positive gMG whose symptoms are difficult to control with high-dose IVIg therapy or PLEX in Japan [18] (Sect. 5). This narrative review focuses on the therapeutic efficacy and tolerability of eculizumab in these patients and summarizes relevant pharmacological data.

\section{Pharmacological Properties of Eculizumab}

The pharmacological properties of eculizumab have been reviewed in detail previously $[19,20]$; a brief summary of data relevant to gMG are discussed here.

\subsection{Mechanism of Action}

Eculizumab is a terminal complement inhibitor comprising human constant regions from human IgG2 and IgG4 sequences and murine complementarity-determining regions grafted onto the human framework light- and heavy-chain variable regions $[16,17]$. It binds with high affinity and specificity to the complement protein C5 [21] and is thought to sterically hinder the $\mathrm{C} 5$ convertases from associating with C5 [22], which inhibits C5 cleavage to the proinflammatory complement component $\mathrm{C} 5 \mathrm{a}$ and $\mathrm{C} 5 \mathrm{~b}$ [21]. Although the exact mechanism is unknown, it is believed that eculizumab exerts its therapeutic effects in patients with gMG by reducing the formation and deposition of the MAC C5b-9 at the NMJ and destruction of the post-synaptic structure (Fig. 1) [16]. Immediate, complete and sustained inhibition of terminal complement activity is seen with chronic administration of eculizumab [17]. Eculizumab blocks the complement cascade at the level of $\mathrm{C} 5$, but preserves the generation of $\mathrm{C} 3 \mathrm{~b}$, which is essential 


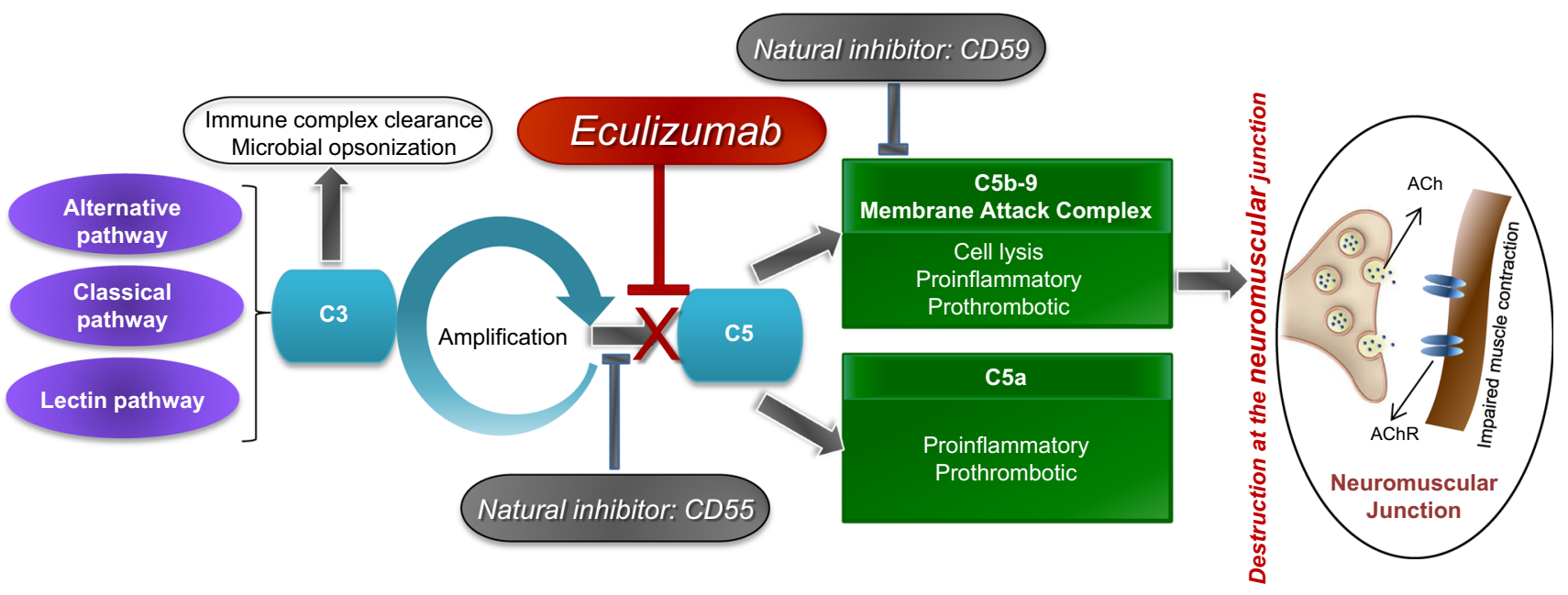

Fig. 1 Mechanism of action of eculizumab. ACh acetylcholine, AChR acetylcholine receptor

for the clearance of circulating immune complexes and the normal phagocytosis of bacterial and fungal pathogens [21]. The clinical efficacy of eculizumab in the pivotal, phase 3 REGAIN trial in patients with refractory gMG is discussed in Sect. 3.

\subsection{Pharmacokinetic Properties}

The pharmacokinetic properties of intravenous eculizumab in patients with refractory gMG were consistent with those in patients with PNH or aHUS [17]. According to a population pharmacokinetic analysis in patients with $\mathrm{gMG}$, eculizumab $900 \mathrm{mg}$ every week for 4 weeks, $1200 \mathrm{mg}$ at week 5 and $1200 \mathrm{mg}$ every 2 weeks thereafter (approved dosage; Sect. 5) was expected to result in eculizumab concentrations above the minimum target $(>50 \mu \mathrm{g} / \mathrm{mL})$ for complete complement blockade in $95 \%$ of patients, thereby reducing the risk of clinical deterioration [23]. With this regimen, the exposure to eculizumab (mean area under the concentration-time curve) was higher than the exposure associated with $50 \%$ of maximal effect [assessed by the quantitative myasthenia gravis (QMG) total score] and close to the plateau of effect [23]. After maintenance doses of $1200 \mathrm{mg}$ every 2 weeks in patients with $\mathrm{gMG}$, the observed mean peak plasma concentration $\left(\mathrm{C}_{\max }\right)$ of eculizumab at week 26 was $783 \mu \mathrm{g} / \mathrm{mL}$ and the mean trough concentration was $341 \mu \mathrm{g} / \mathrm{mL}$ [16]. In all indications, steady state was reached in 4 weeks and the accumulation ratio was $\approx 2$-fold. For a typical $70 \mathrm{~kg}$ patient, the eculizumab volume of distribution was 5-8 L [16].

Human antibodies, like eculizumab, are largely catabolized by lysosomal enzymes to small peptides and amino acids [17]. The elimination half-life of eculizumab was $\approx 270-375 \mathrm{~h}$ [16]. In patients receiving PLEX or fresh frozen plasma infusion, the clearance of eculizumab was increased by $\approx 250$-fold and the elimination half-life was reduced to $1.26 \mathrm{~h}$ [16]; therefore supplemental eculizumab dosing is recommended in these patients [16, 17]. Age (2 months to 85 years), sex, race and renal impairment did not affect the pharmacokinetics of eculizumab [16]; no studies have assessed the effect of hepatic impairment on the pharmacokinetics of eculizumab [17]. No interaction studies of eculizumab with other medicinal products have been undertaken [17].

\section{Therapeutic Efficacy of Eculizumab}

This section focuses on the efficacy of intravenous eculizumab in patients with anti-AChR antibody-positive refractory $\mathrm{gMG}$, based on results from the randomized, double-blind, multicentre, phase 3 REGAIN study (ECUMG-301) [24] and its ongoing open-label extension (ECUMG-302; abstract and poster) [25]. These studies followed a pilot, phase 2 study in patients with severe, refractory, anti-AChR-positive gMG, which showed that eculizumab was associated with clinically meaningful improvements in muscle strength relative to placebo, suggesting its potential for the treatment of gMG [26].

The REGAIN study included patients aged $\geq 18$ years with confirmed gMG, a positive serological test for antiAChR antibodies, impaired activities of daily living [i.e. myasthenia gravis-activities of daily living (MG-ADL) score of $\geq 6$ ] and class II-IV disease according to the Myasthenia Gravis Foundation of America (MGFA) [24]. Patients were also required to have received:

- $\geq 2$ ISTs for 12 months without symptom control or

- $\geq 1$ IST with IVIg or PLEX, at least four times per year for 12 months, without symptom control.

Key exclusion criteria included a history of thymomas or other thymic neoplasms, thymectomy within 12 months 
Table 1 Efficacy of intravenous eculizumab in patients with anti-acetylcholine receptor antibody-positive refractory generalized myasthenia gravis in the randomized, double-blind, multicentre, phase 3 REGAIN study [27]

\begin{tabular}{|c|c|c|c|c|c|}
\hline \multirow[t]{2}{*}{ Analyses } & \multirow[t]{2}{*}{ Treatment } & \multicolumn{4}{|c|}{ LSM rank ${ }^{\mathrm{a}}$ or LSM change from $\mathrm{BL}^{\mathrm{b}}$ in total scores at week $26^{\mathrm{c}}$} \\
\hline & & MG-ADL & QMG & MGC & MG-QOL15 \\
\hline \multicolumn{6}{|l|}{ Worst-rank ANCOVA } \\
\hline \multirow[t]{3}{*}{ Prespecified } & $\mathrm{ECU}$ & $56.6^{\mathrm{d}}$ & $54.7 *$ & 57.3 & $55.5^{*}$ \\
\hline & PL & $68.3^{\mathrm{d}}$ & 70.7 & 67.7 & 69.7 \\
\hline & & BGD - 11.7 & BGD -16.0 & BGD -10.5 & BGD -14.3 \\
\hline \multirow[t]{3}{*}{ Post hoc sensitivity } & ECU & $54.8^{*}$ & $53.9 * *$ & $56.1 *$ & $54.6^{*}$ \\
\hline & PL & 70.2 & 71.6 & 69.0 & 70.6 \\
\hline & & BGD - 15.4 & BGD - 17.7 & BGD - 12.9 & BGD -16.0 \\
\hline \multicolumn{6}{|l|}{ Repeated-measures model } \\
\hline \multirow[t]{3}{*}{ Prespecified sensitivity analysis with IST as covariate } & ECU & $-4.1 * *$ & $-4.6 * * *$ & $-7.9^{*}$ & $-13.8 * * *$ \\
\hline & PL & -2.3 & -1.7 & -4.6 & -6.7 \\
\hline & & BGD -1.8 & BGD - 2.9 & BGD -3.3 & BGD -7.1 \\
\hline \multirow[t]{3}{*}{ Prespecified sensitivity analysis without IST as covariate } & ECU & $-4.2 * *$ & $-4.6^{* * *}$ & $-8.1^{*}$ & $-12.6^{* * *}$ \\
\hline & PL & -2.3 & -1.6 & -4.8 & -5.4 \\
\hline & & BGD -1.9 & BGD -3.0 & BGD -3.3 & BGD -7.2 \\
\hline
\end{tabular}

Results are for the full analysis set comprising 62 patients receiving ECU and 63 patients receiving PL. With the exception of prespecified worstrank ANCOVA for MG-ADL, $p$ values reported are nominal

$B L$ baseline, $B G D$ between-group difference, $E C U$ eculizumab (900 $\mathrm{mg}$ on day 1 and weeks 1, 2, and 3, $1200 \mathrm{mg}$ at week 4, and $1200 \mathrm{mg}$ every second week thereafter), IST immunosuppressive therapy, $L S M$ least-squares mean, $M G-A D L$ myasthenia gravis-activities of daily living, $M G C$ myasthenia gravis composite, $M G$ - $Q O L 15$ 15-item myasthenia gravis quality of life, $P L$ placebo, $Q M G$ quantitative myasthenia gravis

$* p<0.05$, ** $p<0.01, * * * p \leq 0.001$ vs. PL

${ }^{\mathrm{a} C}$ Change from BL to week 26 in total scores given as LSM rank using a worst-rank ANCOVA

${ }^{b}$ At BL, the mean MG-ADL scores in ECU and PL recipients were 10.5 and 9.9; the mean QMG scores were 17.3 and 16.6; the mean MGC scores were 20.4 and 18.9; and the mean MG-QOL15 scores were 33.6 and 30.7, respectively

${ }^{\mathrm{c}}$ Lower rank or a decrease in total score indicates improvement

${ }^{\mathrm{d}}$ Primary endpoint

of screening, MGFA class I, myasthenic crisis (MGFA class V), treatment with IVIg or PLEX within 4 weeks of randomization or rituximab therapy within 6 months of screening [24].

Patients were randomized to eculizumab at the approved dosage (see Table 1 for dosage details) or placebo for 26 weeks [24]. Those receiving prior therapy with a cholinesterase inhibitor, oral corticosteroid or other ISTs were to continue treatment at the same dose and schedule throughout the study, unless an adjustment was needed due to a compelling medical reason. Owing to the severity of disease, rescue medication (e.g. high-dose corticosteroids, IVIg or PLEX) was permitted at the physician's discretion. All randomized patients were required to have been vaccinated against Neisseria meningitides [24]. Patients who completed REGAIN could continue into the open-label extension study, in which all patients received eculizumab maintenance therapy (1200 mg every 2 weeks) [25]. During the extension, the dose of background immunosuppressants could be modified at the investigator's discretion [27].

At baseline, the majority (66\%) of patients were female, $98 \%$ of patients had previously received $\geq 2$ ISTs and $52 \%$ of patients had received $\geq 3$ ISTs [24]. Patients had a mean age of 47.2 years at first study dose; during the study, $78 \%$ of patients received concomitant corticosteroids and $>80 \%$ of patients received $\geq 1$ other IST (e.g. azathioprine, mycophenolate mofetil, cyclosporine) [24]. The mean disease duration was 9.6 years and approximately $54 \%$ of patients had MGFA class IV or V as their worst classification since diagnosis of MG. Patients in the two treatment groups were generally well balanced in terms of demographics, disease characteristics and medical history [24].

The primary endpoint was the change in MG-ADL total score (validated, patient-reported outcome) from baseline to week 26 measured by worst-rank ANCOVA [24]. In this analysis, patients were ranked from 1 (best outcome) to 125 (worst outcome), with all patients who died, had MG crisis, received rescue medication or discontinued therapy (regardless of the reason for discontinuation) assigned to the lowest ranks [24]. Prespecified secondary endpoints (validated measures) were: (1) change from baseline in the QMG total score (physician-evaluated) as assessed by worst-rank ANCOVA, (2) responder analysis of the MG-ADL score ( $\geq$ 3-point improvement from baseline), (3) responder 
analysis of the QMG score ( $\geq 5$-point improvement from baseline), (4) change from baseline in the Myasthenia Gravis Composite (MGC) total score (patient- and physician-reported) as assessed by worst-rank ANCOVA and (5) change from baseline in the Myasthenia Gravis Quality of Life questionnaire (MG-QOL15) total score (patient-reported) as assessed by worst-rank ANCOVA. The primary and prespecified secondary endpoints were assessed hierarchically (in the abovementioned sequence) at week 26; responder analyses assessed the proportion of patients who did not require rescue medication and met the criteria for improvement in total score [24].

In addition, prespecified sensitivity analyses (including repeated-measures analyses) and post hoc sensitivity worstrank ANCOVA of MG-ADL, QMG, MGC and MGQOL15 were undertaken. In the post hoc worst-rank ANCOVA, patients who discontinued therapy but were improving on the MG-ADL scale were not assigned to the lowest ranks; in this analysis, patients who died, patients who experienced MG crisis, patients who required rescue therapy for MG exacerbation or experienced MG exacerbation without requiring rescue therapy were ranked by time to event [24]. A 2-point reduction from baseline in the MG-ADL score [28], 3-point reductions from baseline in the QMG [29] and MGC [30] scores and a 7- to 8-point reduction from baseline in the MG-QOL15 score [31] are established clinically meaningful improvements [24].

\subsection{REGAIN Study}

In the REGAIN study, although there was no significant difference between eculizumab and placebo recipients for the primary endpoint according to the prespecified primary endpoint analysis, prespecified and post hoc sensitivity analyses indicated that eculizumab was effective in improving symptoms of disease [24]. At week 26, eculizumab and placebo recipients did not differ significantly for the least-squares (LS) mean-ranked change from baseline in the MG-ADL score (primary endpoint; $p=0.0698$; Table 1), as measured by the worst-rank ANCOVA. However, in prespecified sensitivity repeatedmeasures analyses (with or without ISTs as covariate), the LS mean change from baseline to week 26 in the MG-ADL score was significantly greater with eculizumab than placebo (Table 1). Moreover, a post hoc worst-rank ANCOVA of the primary endpoint showed a significant benefit with eculizumab over placebo ( $p=0.0160$; Table 1). In this analysis, three eculizumab recipients who discontinued treatment because of adverse events (Moraxella lacunata bacteraemia, bowel perforation and adenocarcinoma of prostate gland) that did not reflect worsening of MG and did not require rescue therapy, had a clinically meaningful response to therapy (3-, 7-, and 7-point improvement, respectively, in MG-ADL score) and were not assigned to the lowest ranks [24].

Eculizumab also significantly improved muscle strength, as indicated by a greater LS mean change from baseline to week 26 in the QMG total score relative to placebo, as measured by the prespecified worst-rank ANCOVA (nominal $p=0.0129$; Table 1). Similar findings were seen in prespecified sensitivity analyses and post hoc worst-rank ANCOVA of this outcome (Table 1) [24].

In responder analyses based on thresholds above the established clinically important differences, significantly greater proportions of eculizumab than placebo recipients achieved clinically meaningful responses in the MG-ADL total score ( $\geq 3$-point improvement; 60 vs. 40\%; $p=0.0229)$ and the QMG total score ( $\geq 5$-point improvement; 45 vs. $19 \%$; $p=0.0018)$. Greater benefit with eculizumab relative to placebo was also seen for higher thresholds of the MG-ADL total score $(\geq 4$ - to $\geq 8$ point improvement) and QMG total score $(\geq 6$ - to $\geq 10$ point improvement) (all nominal $p<0.05$ ) [24]. In addition, an ad hoc dual responder analysis at week 26 (abstract) showed that three times as many eculizumab as placebo recipients had clinically meaningful improvements in both muscle strength ( $\geq 5$-point improvement in QMG total score) and the ADL ( $\geq 3$-point improvement in MGADL total score) (40 vs. 13\%; nominal $p=0.0004$ ) [32].

As with the prespecified primary endpoint analysis, the change from baseline in the MGC total score (assessing signs and symptoms of disease) using prespecified worst-rank ANCOVA did not differ significantly between eculizumab and placebo recipients; however, prespecified sensitivity analyses and post hoc worst-rank ANCOVA indicated a significant benefit of eculizumab over placebo for this outcome (Table 1) [24]. HR-QOL, as assessed by the MGQOL15 total score, was significantly improved with eculizumab relative to placebo based on prespecified worst-rank ANCOVA, as well as in prespecified sensitivity analyses and post hoc worst-rank ANCOVA (Table 1) [24].

The repeated-measures sensitivity analyses without IST as a covariate showed that in eculizumab relative to placebo recipients, significant improvements in the MG-ADL and MGC total scores were seen by week 1, the QMG total score by week 2 and the MG-QOL15 total score when assessed at week 4 of treatment (all $p<0.05$ ) [24]. The maximum treatment effect was generally evident by week 12 , with the benefits sustained over 26 weeks' therapy [24].

REGAIN also assessed the effect of eculizumab on fatigue, using the patient-reported Neuro-QOL Fatigue scale, which is validated in other neurological disorders (abstract) [33]. At week 26, eculizumab recipients had greater improvement in fatigue than placebo recipients, based on the repeated-measures sensitivity analysis (mean change from baseline -16.8 vs. $-7.9 ; p=0.0061$ ) [33]. 
In addition, an analysis of correlation between efficacy outcomes showed that Neuro-QOL Fatigue had a strong positive correlation with MG-ADL and MG-QOL15 (correlation coefficient $\mathrm{r}=0.63$ and 0.75 , respectively; both $p<0.0001)$ and a moderate positive correlation with QMG $(\mathrm{r}=0.57 ; p<0.0001)$ (abstract) [34].

\subsection{Extension Study}

The ongoing extension of REGAIN $(n=116)$ showed that the treatment effect of eculizumab is sustained during at least 52 weeks' of continued therapy (abstract) [25]. In patients who received eculizumab therapy throughout (i.e. in REGAIN and its extension; $n=56$ ), the MG-ADL score (primary efficacy endpoint) remained largely unchanged at each assessment from baseline (i.e. start of the extension study) to week 52. In patients who switched from placebo in REGAIN to eculizumab in the extension $(n=60)$, a significant improvement from baseline in the MG-ADL score was seen as early as week 1 of therapy $(-1.6$; $p<0.0001$ ). Most of the treatment effect in these patients was seen by week $8(-2.6 ; p<0.0001)$, with benefit maintained up to at least 52 weeks $(-2.7 ; p<0.0001)$. Generally similar response patterns were seen on the respiratory, bulbar, limb and ocular domains of MG-ADL [25], and for the QMG, MGC and MG-QOL15 total scores (abstract) [35]. During the extension, the daily dose of one background IST was reduced in $47 \%$ of patients overall (the most common reason being improvement in MG symptoms with eculizumab) and $16.2 \%$ of patients stopped IST, suggesting a steroid-sparing effect of eculizumab [27]. The daily dose of one IST was increased in $24.8 \%$ of patients overall and $4.3 \%$ of patients started new IST [27].

\section{Tolerability of Eculizumab}

Intravenous eculizumab was generally well tolerated in patients with refractory gMG, with a tolerability profile generally similar to that seen previously in other indications [24]. Across 10 disease populations (total $n=1407$ patients), including $\mathrm{PNH}$, aHUS and refractory gMG, the most common adverse reaction associated with eculizumab was headache (incidence $\geq 1 / 10$ ), which occurred largely during the initial phase of treatment [17]; like gMG, PNH and aHUS are also treated with immunosuppressants. In terms of serious adverse reactions, of all meningococcal infections with eculizumab, meningococcal sepsis was reported most commonly [17]. Owing to its mechanism of action (Sect. 2), eculizumab may increase patients' susceptibility to meningococcal infections, and life-threatening and fatal meningococcal infections have been reported in patients receiving eculizumab therapy [16, 17].
Consequently, patients should be monitored for early signs of meningococcal infection and evaluated immediately if infection is suspected [16, 17]; if necessary, patients should be treated with appropriate antibiotics [17]. The risk of other systemic infections (particularly with encapsulated bacteria) may also be increased with eculizumab treatment; therefore, eculizumab should be administered with caution in patients with active systemic infections $[16,17]$.

In the 26-week REGAIN study in patients with refractory $\mathrm{gMG}$, the overall incidence of treatment-emergent adverse events (AEs) in eculizumab and placebo recipients were generally similar ( 86 vs. $89.0 \%$ ), with most AEs being of mild or moderate severity; 48 and $40 \%$ of AEs, respectively, were considered related to the drug [27]. The most common ( $\geq 10 \%$ in either group) treatment-emergent AEs in the eculizumab and placebo groups were headache (16 vs. 19\%), upper respiratory tract infection (URTI; 16 vs. $19 \%)$, nasopharyngitis (15 vs. $16 \%$ ), nausea (13 vs. $14 \%$ ), diarrhoea (13 vs. $13 \%$ ) and MG (10 vs. $17 \%$ ) [24]. Treatment-emergent AEs led to discontinuation of therapy in four eculizumab recipients (including one patient who had MG crisis and died 90 days after the last eculizumab dose because of crisis-related complications) compared with no placebo recipient [24].

Serious treatment-emergent AEs occurred in $15 \%$ of eculizumab and $29 \%$ of placebo recipients, with infections reported most frequently [four events in two patients (3\%) and seven events in six patients (10\%), respectively] [24]. Approximately half as many eculizumab as placebo recipients required rescue therapy (10 vs. 19\%) or reported MG exacerbations ( 10 vs. $24 \%$ ); three patients (all in the placebo group) who had exacerbations did not require rescue therapy. There were no deaths or cases of meningococcal infections during the study [24]. As with all proteins, there is a potential for immunogenicity with eculizumab, and antibody responses have been detected infrequently across all clinical studies; no eculizumab recipient in REGAIN showed an antidrug antibody response [16, 17]. No patient in REGAIN experienced an infusion reaction that required discontinuation of eculizumab treatment $[16,17]$.

The tolerability profile of eculizumab during longerterm treatment ( $\geq 52$ weeks) in the extension study was generally similar to that during short-term therapy, with the most common (incidence $>10 \%$ ) AEs being headache (26.5\%), nasopharyngitis (23.9\%), diarrhoea (14.5\%), MG $(14.5 \%)$, arthralgia (12.0\%) and URTI (11.1\%) [25].

\section{Dosage and Administration of Eculizumab}

Intravenous eculizumab is indicated for the treatment of gMG in the USA [16] and refractory gMG in the EU [17] in adults who are anti-AChR antibody-positive. It is also 
approved in Japan for use in patients with gMG who are anti-AChR antibody-positive and whose symptoms are difficult to control with high-dose IVIg or PLEX [18]. The recommended dosage of eculizumab is $900 \mathrm{mg}$ every week for 4 weeks (initial phase) followed by $1200 \mathrm{mg}$ for the fifth week and $1200 \mathrm{mg}$ every 2 weeks thereafter (maintenance phase). Eculizumab should be administered as a $35 \mathrm{~min}$ (in the USA) or a $25-45 \mathrm{~min}$ (in the EU) intravenous infusion; one single-dose vial of $30 \mathrm{~mL}$ contains $300 \mathrm{mg}$ of eculizumab [16, 17]. Supplemental dosing of eculizumab is required in patients receiving concomitant plasmapheresis or PLEX, or fresh frozen plasma infusion (see local prescribing information for further details).

There is a risk of serious meningococcal infections with eculizumab therapy [16, 17] (Sect. 4) and the US prescribing information carries a boxed warning regarding the same [16]. Eculizumab is contraindicated in patients with unresolved serious $N$. meningitides infection [16, 17] and in patients who are not currently vaccinated against $N$ meningitides, unless the risks of delaying treatment outweigh the risks of developing a meningococcal infection (USA) [16] or patients receive prophylactic treatment with appropriate antibiotics until 2 weeks after vaccination (EU) [17]. Local prescribing information should be consulted for further information, including dosage and administration details, contraindications, warnings and precautions.

\section{Current Status of Eculizumab in Generalized Myasthenia Gravis}

According to the international consensus guidelines, the aim of treatment in $\mathrm{MG}$ is to achieve at least Minimal Manifestation Status (i.e. no symptoms or functional limitations from MG but have some weakness on examination of some muscles) with no more than grade 1 AEs [12]. The acetylcholineesterase inhibitor pyridostigmine is recommended as part of initial treatment in most patients with MG. Patients who do not achieve treatment goals with pyridostigmine should receive corticosteroids or IST, and when corticosteroids are contraindicated or refused, a nonsteroidal immunosuppressant should be used. For patients with refractory $\mathrm{MG}$, in addition to the abovementioned treatment options, chronic PLEX, chronic IVIg, cyclophosphamide and rituximab are recommended by the international consensus guidelines. Chronic PLEX and chronic IVIg are also recommended for the management of myasthenic crises [12]. The Association of British Neurologists [36] and German Neurological Society [2] treatment guidelines have generally similar recommendations for the management of MG.

Research in recent years has focused on identifying new targets and developing novel therapies, particularly for patients with MG who are refractory to conventional therapies. These patients have unchanged or worse status after treatment with corticosteroids and at least two other immunosuppressive agents (used in adequate doses for an adequate duration) with persistent symptoms or side effects that limit functioning, as defined by patient and physician [12]. Patients with refractory disease continue to have symptoms, which adversely affect the ADL, and may experience frequent exacerbations, which can be lifethreatening and require hospital care [24]. Eculizumab is one such novel therapy; it is the first targeted complement inhibitor approved for use in anti-AChR antibody-positive adults with gMG (USA) [16], refractory gMG (EU) [17] or gMG with symptoms that are difficult to control with highdose IVIg or PLEX (Japan) [18]. Although treatment guidelines for $\mathrm{MG}$ were updated prior to the approval of eculizumab, current German Neurological Society guidelines include eculizumab as an option for use in patients with severe, refractory gMG [2].

Eculizumab is a recombinant humanized monoclonal antibody that binds to human C5 complement protein and inhibits the activation of terminal complement, thereby protecting the NMJ from the destructive effects of antibody-mediated complement activation (Sect. 2). The clinical benefit of treatment with eculizumab was demonstrated in the well-designed, 26-week REGAIN study in patients with anti-AChR-positive refractory gMG [24], with treatment benefits sustained during the 52-week extension study [25] (Sect. 3). Although the prespecified primary endpoint analysis for the change from baseline to week 26 in the MG-ADL score as measured by worst-rank ANCOVA did not show statistically significant difference between eculizumab and placebo recipients, prespecified and post hoc sensitivity analyses showed significant improvements in the ADL with eculizumab relative to placebo, regardless of background IST (Sect. 3.1). Prespecified sensitivity and secondary analyses were included in the analysis plan to assess the treatment effect comprehensively (reflecting both the patients' and physicians' perspective), as the use of the worst-rank analysis would have made it difficult to generalize the results to clinical practice [24].

Failure to meet the primary endpoint appears to be related to the worst-rank analytical approach used for assessment, according to which all patients who discontinued therapy were assigned to the poor outcome group, regardless of the reason for discontinuation [24, 27]. Significant treatment benefit seen in the post hoc analysis of the primary outcome illustrated the importance of differentiating between patients with poor outcomes related to MG and those with poor outcomes unrelated to MG at the time of treatment discontinuation [24]. In addition, the sample size of the study may have been inadequate for the use of the worst-rank analytical approach [24]. 
Other outcomes, including muscle strength (QMG score) and HR-QOL measures (MG-QOL15 and NeuroQOL Fatigue scores) improved significantly with eculizumab relative to placebo, supporting the efficacy of eculizumab in patients with anti-AChR-positive refractory gMG (Sect. 3.1). Responder analyses (a particularly important outcome in rare diseases [24]) from REGAIN showed that significantly more eculizumab than placebo recipients had clinically meaningful improvements (based on thresholds above the established criteria) in MG-ADL and QMG scores (Sect. 3.1). Up to $40 \%$ of placebo recipients also had clinically meaningful improvement in these outcomes (Sect. 3.1), which, according to the study authors, may reflect the known effect of responsiveness with placebo in patients with neurological symptoms, variability of myasthenic symptoms in the short term or greater potential for variability in patients with refractory disease [24].

Immunomodulatory therapies, such as IVIg and PLEX, act rapidly, while ISTs targeting autoantibody production (including commonly used agents that are considered steroid-sparing) may require several months to provide clinical benefit [37]. Eculizumab also acts rapidly, with treatment benefits seen within 4 weeks of initiating treatment in REGAIN, maximal effects generally seen within 12 weeks (Sect. 3.1) and clinical benefits maintained during the ongoing 52-week extension (Sect. 3.2) [24]. However, the overall duration for which eculizumab treatment may be required remains to be determined [38] and may depend on the mechanism of disease. Further research is needed to determine if the formation of MAC and destruction of the NMJ would restart after discontinuation of complement inhibition.

Eculizumab itself appears to have a steroid-sparing effect, as evidenced by a greater proportion of eculizumab recipients reducing their daily dose of IST or stopping IST than those increasing their daily dose of IST or starting new IST (63 vs. 29\%; Sect. 3.2). However, as eculizumab does not target the autoantibody component of the disease, it is unlikely that patients with gMG will be able to discontinue all other therapies.

Eculizumab was generally well tolerated in patients with anti-AChR-positive refractory gMG during $\geq 78$ weeks' therapy in REGAIN and its extension, with a tolerability profile generally similar to that seen previously in other indications and no new safety concerns identified (Sect. 4). The tolerability and efficacy of eculizumab during longterm therapy remains to be established, and final results from the extension of REGAIN are awaited with interest.

Although the REGAIN study did not include patients with thymoma, treatment with eculizumab is expected to be beneficial in these patients, as their autoantibody- profile is similar to that of the REGAIN study population [38]; confirmatory studies in this subgroup of MG patients would be helpful, although patient numbers could be limiting. By contrast, eculizumab is not expected to be effective in patients with anti-MuSK IgG4 antibody-positive MG, as this IgG subclass inhibits complement activation [38].

In addition to the efficacy and tolerability, cost-effectiveness is an important consideration when choosing a therapy. Currently there are no pharmacoeconomic analyses of eculizumab in patients with gMG; well-designed studies assessing the cost-effectiveness of eculizumab are needed, especially considering the high acquisition cost [39] of eculizumab.

To conclude, eculizumab is the first targeted complement inhibitor to be approved for use in anti-AChR antibody-positive adults with gMG or refractory gMG. In the well-designed REGAIN study, although a statistically significant benefit of eculizumab over placebo in the prespecified primary endpoint analysis was not formally demonstrated, preplanned and post hoc sensitivity analyses of this outcome and other secondary outcomes, as well as data from an extension study supported the efficacy of eculizumab in this indication. Eculizumab was generally well tolerated, with a tolerability profile generally similar to that reported previously in other indications. Although several questions remain, such as duration of treatment, cost effectiveness and long-term efficacy and tolerability, current evidence indicates that eculizumab is a valuable emerging therapy for patients with refractory $\mathrm{gMG}$.

\section{Data Selection Eculizumab: 287 records identified}

Duplicates removed

Excluded during initial screening (e.g. press releases; news reports; not relevant drug/indication; preclinical study; reviews; case reports; not randomized trial)

Excluded during writing (e.g. reviews; duplicate data; small patient number; nonrandomized/phase I/II trials)

Cited efficacy/tolerability articles

Search Strategy: EMBASE, MEDLINE and PubMed from 1946 to present. Clinical trial registries/databases and websites were also searched for relevant data. Key words were Eculizumab, Soliris, 5G1.1, myasthenia gravis, gMG. Records were limited to those in English language. Searches last updated 26 January 2018

Acknowledgements During the peer review process, the manufacturer of eculizumab (Soliris ${ }^{\circledR}$ ) was also offered an opportunity to review this article. Changes resulting from comments received were made on the basis of scientific and editorial merit. 


\section{Compliance with Ethical Standards}

Funding The preparation of this review was not supported by any external funding.

Conflict of interest Sohita Dhillon is a salaried employee of Adis/ Springer, is responsible for the article content and declares no relevant conflicts of interest.

Additional information about this Adis Drug Review can be found at http://www.medengine.com/Redeem/39FCF0602D6721C2.

\section{References}

1. Tuzun E, Huda R, Christadoss P. Complement and cytokine based therapeutic strategies in myasthenia gravis. J Autoimmun. 2011;37(2):136-43.

2. Melzer N, Ruck T, Fuhr P, et al. Clinical features, pathogenesis, and treatment of myasthenia gravis: a supplement to the guidelines of the German Neurological Society. J Neurol. 2016;263(8):1473-94.

3. Carr AS, Cardwell CR, McCarron PO, et al. A systematic review of population based epidemiological studies in myasthenia gravis. BMC Neurol. 2010;10:46.

4. Berrih-Aknin S, Le Panse R. Myasthenia gravis: a comprehensive review of immune dysregulation and etiological mechanisms. J Autoimmun. 2014;52:90-100.

5. Meriggioli MN, Sanders DB. Muscle autoantibodies in myasthenia gravis: beyond diagnosis? Expert Rev Clin Immunol. 2012;8(5):427-38.

6. National Institute of Neurological Disorders and Stroke. Myasthenia gravis fact sheet. 2017. https://www.ninds.nih.gov/ Disorders/Patient-Caregiver-Education/Fact-Sheets/MyastheniaGravis-Fact-Sheet\#3. Accessed 8 Jan 2018.

7. Sieb JP. Myasthenia gravis: an update for the clinician. Clin Exp Immunol. 2014;175(3):408-18.

8. Huda R, Tuzun E, Christadoss P. Targeting complement system to treat myasthenia gravis. Rev Neurosci. 2014;25(4):575-83.

9. Boldingh MI, Dekker L, Maniaol AH, et al. An up-date on healthrelated quality of life in myasthenia gravis: results from population based cohorts. Health Qual Life Outcomes. 2015;13:115.

10. Silvestri NJ, Wolfe GI. Treatment-refractory myasthenia gravis. J Clin Neuromuscul Dis. 2014;15(4):167-78.

11. Suh J, Goldstein JM, Nowak RJ. Clinical characteristics of refractory myasthenia gravis patients. Yale $\mathrm{J}$ Biol Med. 2013;86(2):255-60.

12. Sanders DB, Wolfe GI, Benatar M, et al. International consensus guidance for management of myasthenia gravis: executive summary. Neurology. 2016;87(4):419-25.

13. Engel AG, Lambert EH, Howard FM. Immune complexes (IgG and $\mathrm{C} 3$ ) at the motor end-plate in myasthenia gravis: ultrastructural and light microscopic localization and electrophysiologic correlations. Mayo Clin Proc. 1977;52(5):267-80.

14. Nakano S, Engel AG. Myasthenia gravis: quantitative immunocytochemical analysis of inflammatory cells and detection of complement membrane attack complex at the end-plate in 30 patients. Neurology. 1993;43(6):1167-72.

15. Zhou Y, Gong B, Lin F, et al. Anti-C5 antibody treatment ameliorates weakness in experimentally acquired myasthenia gravis. J Immunol. 2007;179(12):8562-7.

16. Alexion Pharmaceuticals Inc. Soliris (eculizumab): US prescribing information. 2015. https://www.accessdata.fda.gov/drugsatfda_ docs/label/2017/125166s422lbl.pdf. Accessed 10 Nov 2017.

17. Alexion Europe SAS. Soliris (eculizumab): summary of product characteristics. 2017. http://www.ema.europa.eu/docs/en_GB/
document_library/EPAR_-_Product_Information/human/000791/ WC500054208.pdf. Accessed 11 Nov 2017.

18. Japan Ministry of Health Labour and Welfare. Soliris (eculizumab): Japanese prescribing information. 2017. http://wwwhourei. mhlw.go.jp/hourei/doc/tsuchi/T171226I0050.pdf. Accessed 10 Jan 2018.

19. Keating GM. Eculizumab: a review of its use in atypical haemolytic uraemic syndrome. Drugs. 2013;73(18):2053-66.

20. McKeage K. Eculizumab: a review of its use in paroxysmal nocturnal haemoglobinuria. Drugs. 2011;71(17):2327-45.

21. Thomas TC, Rollins SA, Rother RP, et al. Inhibition of complement activity by humanized anti-C5 antibody and single-chain Fv. Mol Immunol. 1996;33(17-18):1389-401.

22. Schatz-Jakobsen JA, Zhang Y, Johnson K, et al. Structural basis for eculizumab-mediated inhibition of the complement terminal pathway. J Immunol. 2016;197(1):337-44.

23. Lathia C, Gao X, Kassir N, et al. Population pharmacokinetic and pharmacodynamic analysis of eculizumab to support phase III dosing regimen in patients with refractory generalized myasthenia gravis [abstract no. PII-091]. Clin Pharmacol Ther. 2015;97(Suppl 1):S87.

24. Howard JF, Utsugisawa K, Benatar M, et al. Safety and efficacy of eculizumab in anti-acetylcholine receptor antibody-positive refractory generalised myasthenia gravis (REGAIN): a phase 3, randomised, double-blind, placebo-controlled, multicentre study. Lancet Neurol. 2017. https://doi.org/10.1016/S14744422(17)30369-1.

25. Howard J, Wang JJ, O'Brien F, et al. Efficacy of eculizumab on myasthenia gravis-activities of daily living and its respiratory, bulbar, limb and ocular domains in patients with ACHR+ refractory generalized myasthenia gravis [abstract no. 210 and poster]. Muscle Nerve. 2017;56(3):106.

26. Howard JF Jr, Barohn RJ, Cutter GR, et al. A randomized, double-blind, placebo-controlled phase II study of eculizumab in patients with refractory generalized myasthenia gravis. Muscle Nerve. 2013;48(1):76-84.

27. European Medicines Agency. Soliris (eculizumab): CHMP extension of indication variation assessment report. 2017. http:// www.ema.europa.eu/docs/en_GB/document_library/EPAR_-_ Assessment_Report___Variation/human/000791/WC500233876. pdf. Accessed 10 Nov 2017.

28. Muppidi S. The myasthenia gravis: specific activities of daily living profile. Ann NY Acad Sci. 2012;1274:114-9.

29. Katzberg HD, Barnett C, Merkies IS, et al. Minimal clinically important difference in myasthenia gravis: outcomes from a randomized trial. Muscle Nerve. 2014;49(5):661-5.

30. Burns TM. The MG composite: an outcome measure for myasthenia gravis for use in clinical trials and everyday practice. Ann NY Acad Sci. 2012;1274:99-106.

31. Burns TM, Grouse CK, Conaway MR, et al. Construct and concurrent validation of the MG-QOL15 in the practice setting. Muscle Nerve. 2010;41(2):219-26.

32. Howard J, O'Brien F, Wang JJ, et al. Dual responder analyses of both muscle strength and activities of daily living, eculizumab versus placebo, in refractory generalized myasthenia gravis (gMG) patients: results from the REGAIN study [abstract no. 012]. Neurology. 2017;88(19 Suppl).

33. Andersen H, Mantegazza R, Derosier F, et al. Eculizumab improves fatigue in patients with refractory generalized myasthenia gravis in the REGAIN study [abstract no. PR1094]. Eur J Neurol. 2017;24(Supp 1):498.

34. Andersen H, Mantegazza R, Derosier F, et al. Correlation of neuro-QOL with MG-ADL, QMG, and MG-QOL15 in assessing the spectrum of disease in patients with refractory generalised myasthenia gravis in the REGAIN study [abstract no. PR1095]. Eur J Neurol. 2017;24(Supp 1):498-9. 
35. Howard J, Wang JJ, O'Brien F, et al. Efficacy of eculizumab is maintained beyond 26 weeks in patients with AChR+ refractory generalized myasthenia gravis [abstract no. 211 plus poster]. Muscle Nerve. 2017;56(3):106.

36. Sussman J, Farrugia ME, Maddison P, et al. Myasthenia gravis: Association of British Neurologists' management guidelines. Pract Neurol. 2015;15(3):199-206.

37. Guptill JT, Soni M, Meriggioli MN. Current treatment, emerging translational therapies, and new therapeutic targets for autoimmune myasthenia gravis. Neurotherapeutics. 2016;13(1):118-31.
38. Gilhus NE. Eculizumab: a treatment option for mysthenia gravis? Lancet Neurol. 2017. https://doi.org/10.1016/S14744422(17)30363-0.

39. Medicines Complete. Eculizumab. 2017. https://www.medicines complete.com/mc/bnf/current/DMD11812511000001108.htm?q= eculizumab\&t=search \&ss=text $\&$ tot $=2 \& \mathrm{p}=2 \#$ DMD 11812511000 001108. Accessed 24 Nov 2017. 